Supporting Information for

\title{
Tribenzyl(methyl)ammonium: a Versatile Counterion for the Crystallization of Nanojars with Incarcerated Selenite and Phosphite Ions, and Tethered Pyrazole Ligands
}

\author{
Wisam A. Al Isawi, ${ }^{a}$ Matthias Zeller ${ }^{\mathrm{b}}$ and Gellert Mezei ${ }^{\mathrm{a} *}$ \\ ${ }^{a}$ Department of Chemistry, Western Michigan University, Kalamazoo, Michigan 49008, USA \\ ${ }^{b}$ Department of Chemistry, Purdue University, West Lafayette, Indiana 47907, USA \\ ${ }^{*}$ Corresponding author. Email: gellert.mezei@wmich.edu
}

\section{CONTENTS}

PAGE

1. ESI-MS isotopic distributions for nanojars (Figure S1)

S2

2. Thermal ellipsoid plots of (TBMA) $\mathrm{NO}_{3}, \mathbf{1}, \mathbf{2}$ and $\mathbf{3}$ (Figures S2-S5)

S3-S5

3. Packing diagrams of (TBMA) $\mathrm{NO}_{3}, \mathbf{2}$ and $\mathbf{3}$ (Figures S6-S8)

S6-S8

4. Crystallographic data (Tables S1-S7)

S9-S15 

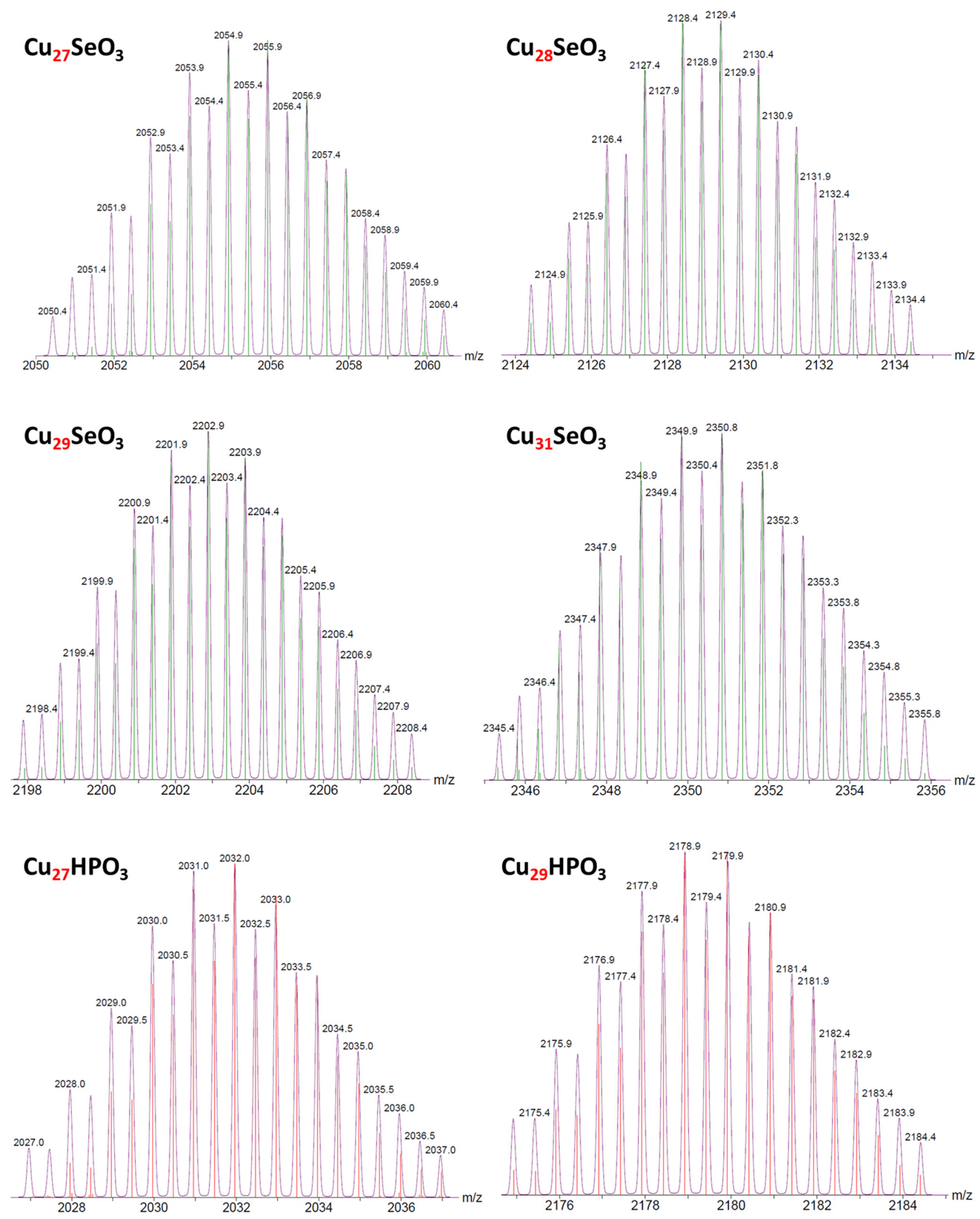

Figure S1. Isotopic distributions observed (centroid) and predicted (continuum) for the selenite and phosphite nanojars. 


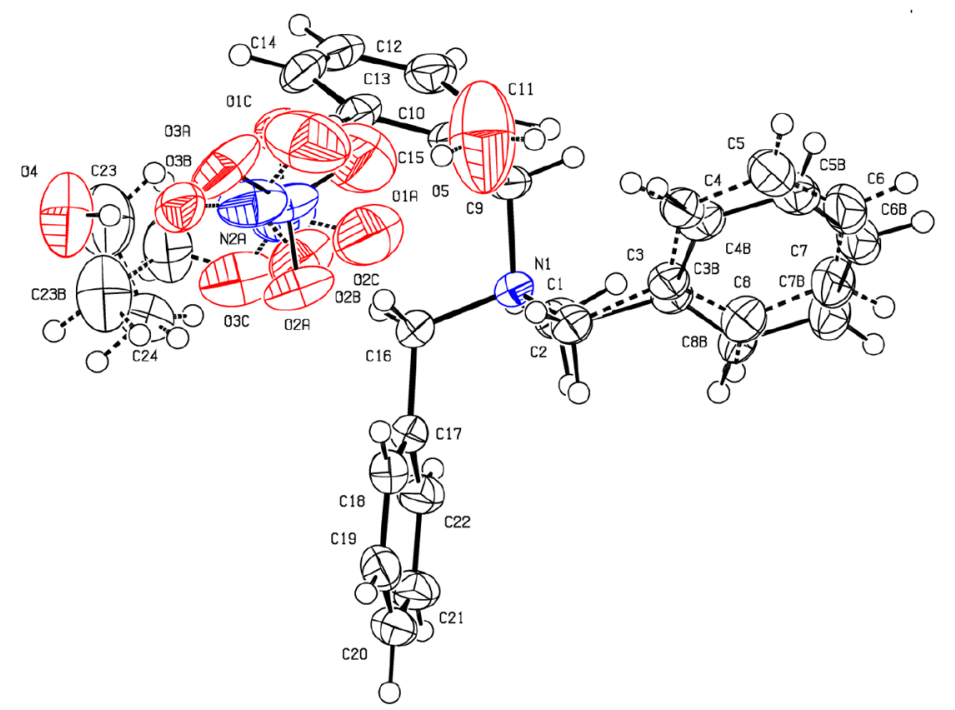

Figure S2. Thermal ellipsoid plot of the crystal structure of (TBMA) $\mathrm{NO}_{3} \cdot$ ethanol $\cdot \mathrm{H}_{2} \mathrm{O}$.
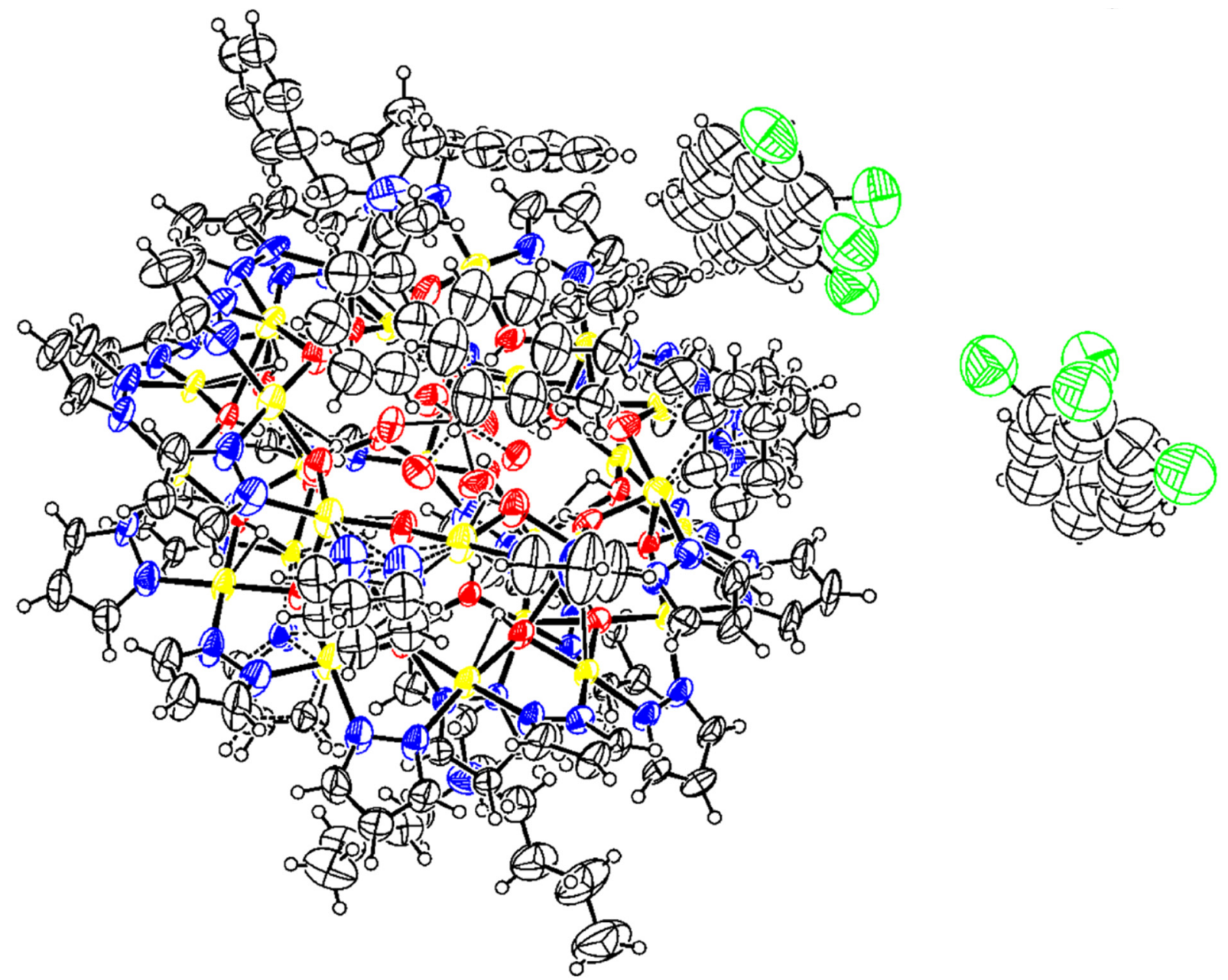

Figure S3. Thermal ellipsoid plot of the crystal structure of $\mathbf{1}$-solvent (solvent $=1,2$-dichlorobenzene, benzonitrile and hexane). 


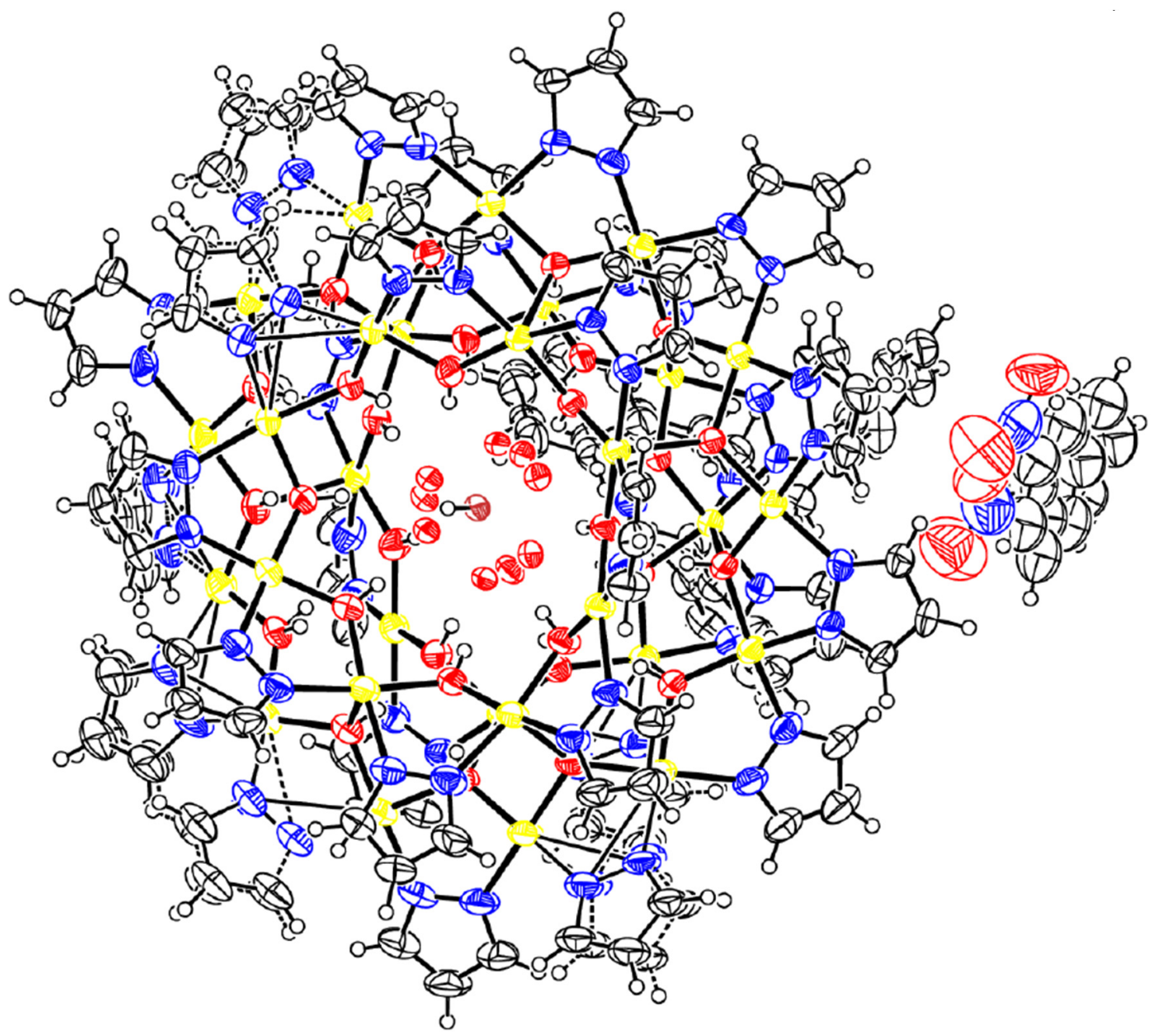

Figure S4. Thermal ellipsoid plot of the crystal structure of $\mathbf{2}$-solvent (solvent $=$ 1,2-dichlorobenzene, benzonitrile and hexane). 


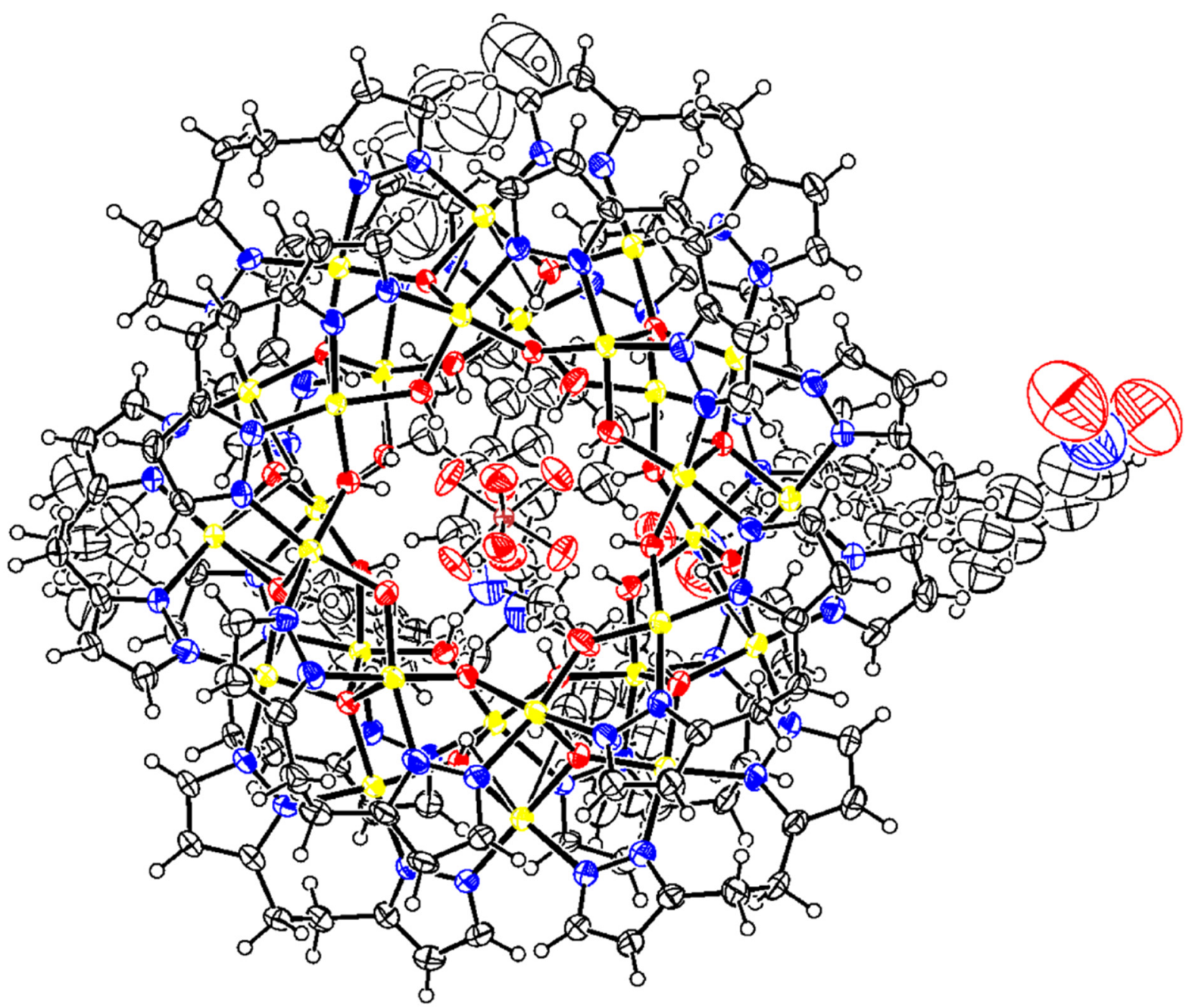

Figure S5. Thermal ellipsoid plot of the crystal structure of $\mathbf{3} \cdot$ solvent (solvent $=1$,2-dichlorobenzene, benzonitrile and hexane). 


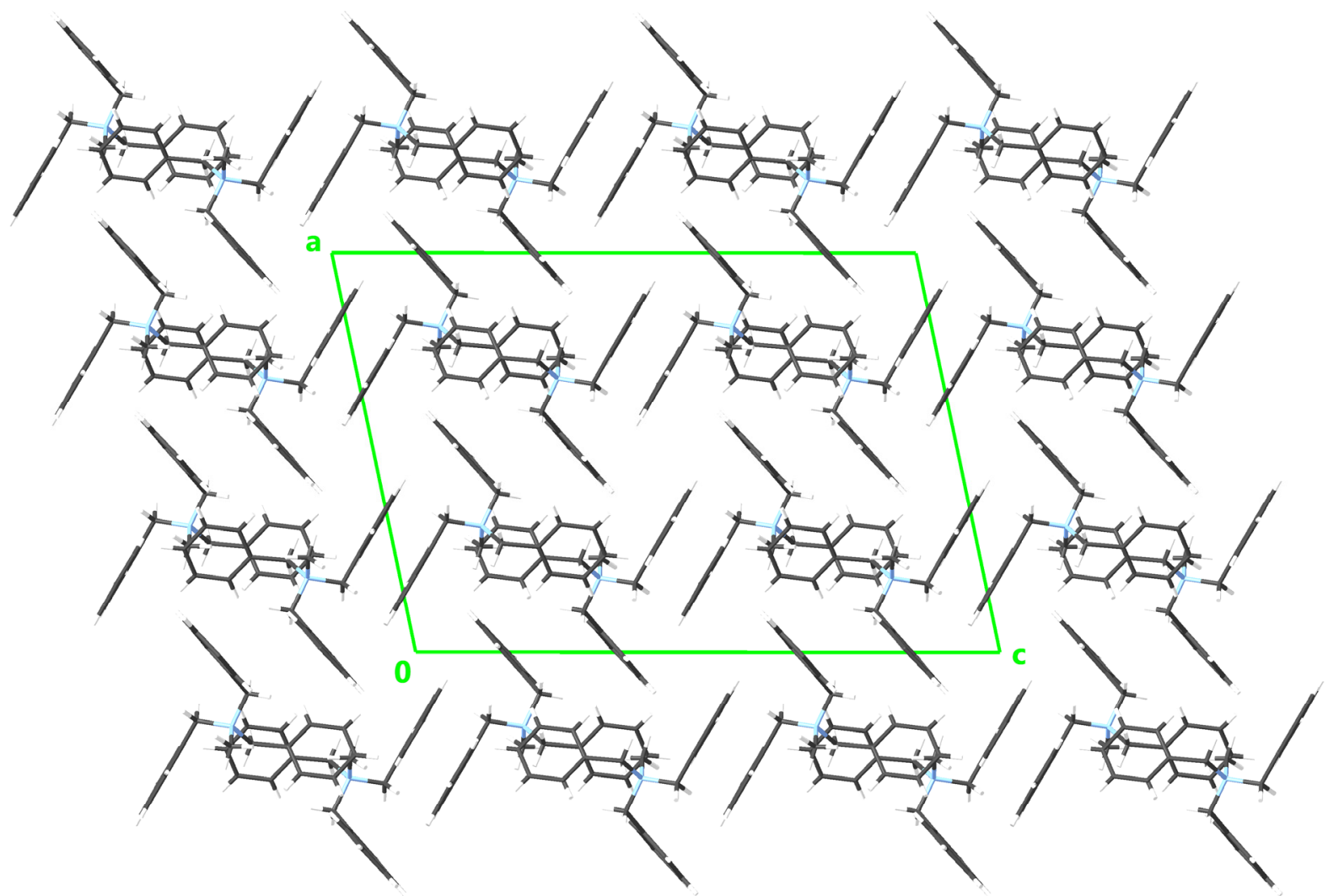

Figure S6. Packing diagram of (TBMA) $\mathrm{NO}_{3}$ along the $b$ axis. The disordered counterion and lattice solvent molecules are omitted for clarity, and only one component is shown for the disordered phenyl moiety. 

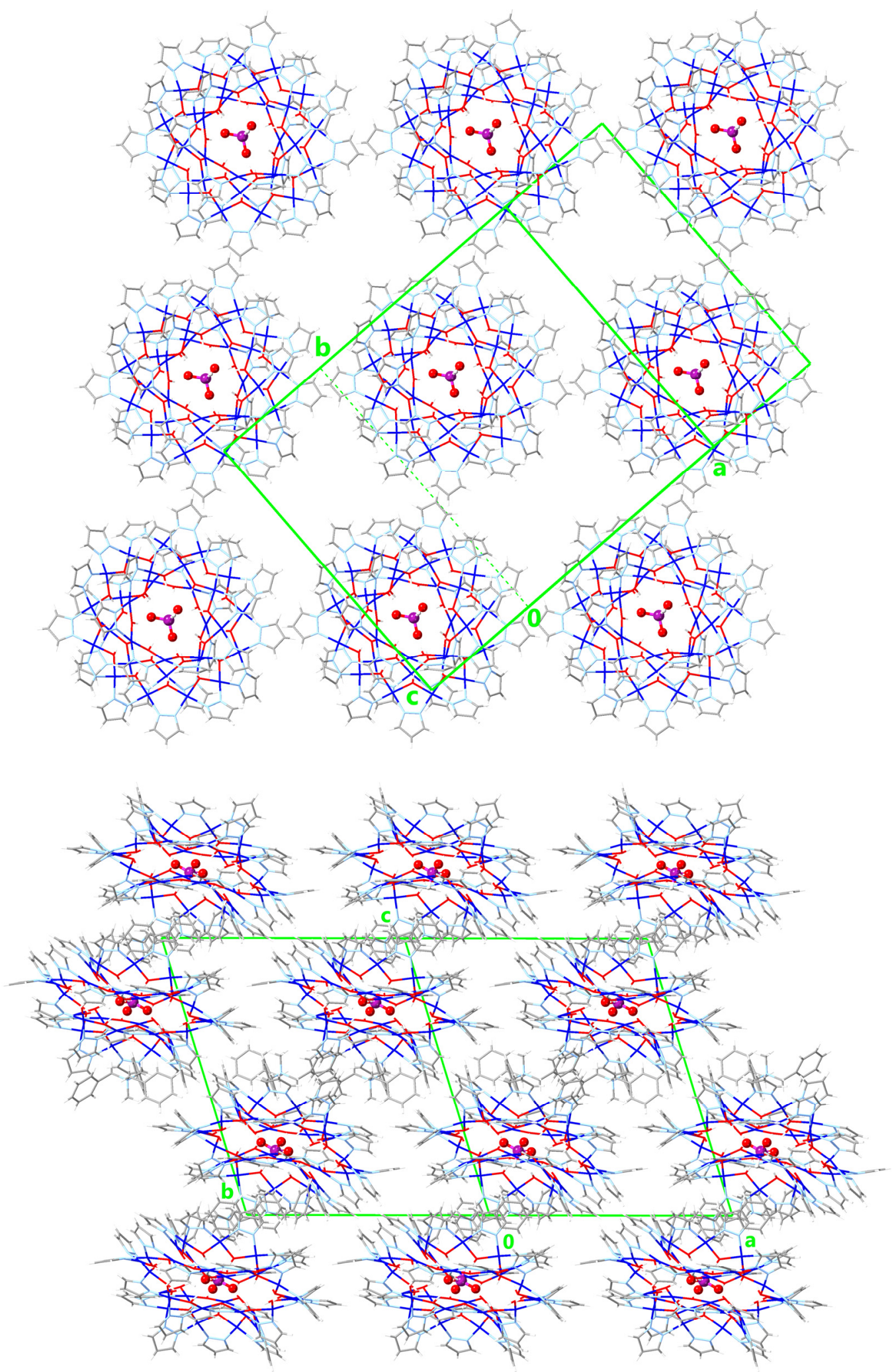

Figure S7. Packing diagrams of $\mathbf{2}$ showing top- (upper; only one plane shown, without counterions) and side-views (lower) of nanojar packing. Lattice solvent molecules are omitted for clarity, and only one component is shown for disordered phosphite and pyrazolate moieties. 

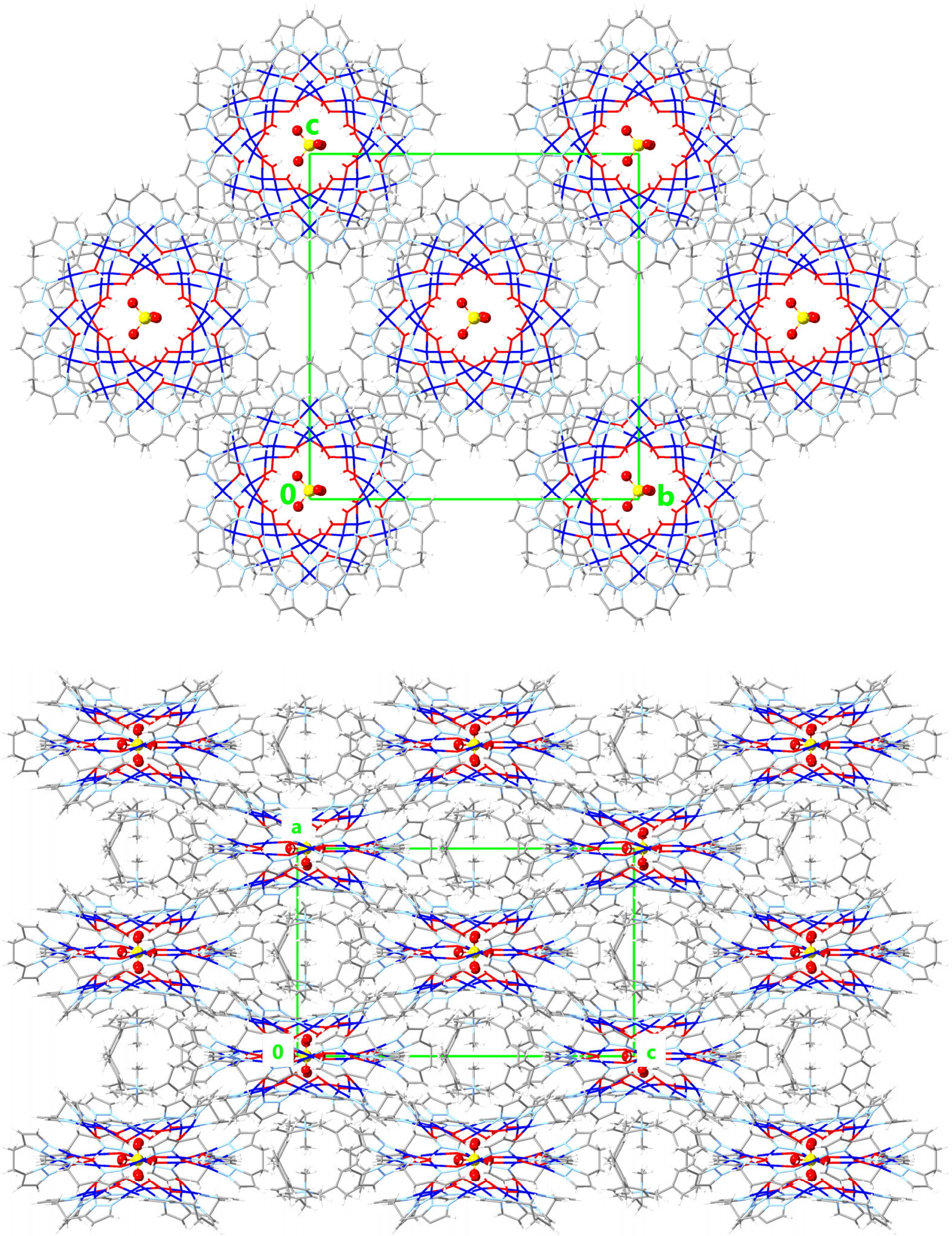

Figure S8. Packing diagrams of $\mathbf{3}$ showing views down the $b$ (upper; without counterions) and $a$ axes (lower). Lattice solvent molecules are omitted for clarity, and only one component is shown for disordered sulfate and pyrazolate moieties. 
Table S1. Comparison of bond lengths, $\mathrm{Cu} \cdots \mathrm{O}$ interactions $(<2.920(1) \AA)$ and hydrogen bonding (donoracceptor $\mathrm{O} \cdots \mathrm{O}$ distance $<3.000(1) \AA$ ) in $\mathbf{1}, \mathbf{2}$ and $\mathbf{3}$ (in $\AA$ ).

\begin{tabular}{|c|c|c|c|}
\hline & 1 & 2 & 3 \\
\hline $\mathrm{Cu}-\mathrm{O}$ bond-length range within $\mathrm{Cu}_{\mathrm{n}}$-rings & $1.885(5)-1.958(4)$ & $1.886(4)-1.945(4)$ & $1.884(7)-1.958(7)$ \\
\hline $\mathrm{Cu}-\mathrm{N}$ bond-length range within $\mathrm{Cu}_{\mathrm{n}}$-rings & $1.85(2)-2.08(1)$ & $1.87(2)-2.027(5)$ & $1.953(8)-2.020(8)$ \\
\hline $\begin{array}{l}\mathrm{Cu}^{\cdots} \mathrm{O} \text { interaction range between } \mathrm{Cu}_{6}-\text { and } \\
\mathrm{Cu}_{12} \text {-rings (1) }\end{array}$ & $2.306(4)-2.481(4)$ & - & - \\
\hline $\begin{array}{l}\text { Average of six } \mathrm{Cu}^{\cdots} \cdots \mathrm{O} \text { interactions between } \\
\mathrm{Cu}_{6^{-}} \text {and } \mathrm{Cu}_{12} \text {-rings }\end{array}$ & $2.395(4)$ & - & - \\
\hline $\begin{array}{l}\mathrm{Cu}^{\cdots} \mathrm{O} \text { interaction range between } \mathrm{Cu}_{10} \text { and } \\
\mathrm{Cu}_{12} \text {-rings }\end{array}$ & $2.364(5)-2.778(5)$ & - & - \\
\hline $\begin{array}{l}\text { Average of six } \mathrm{Cu}^{\cdots} \cdots \mathrm{O} \text { interactions between } \\
\mathrm{Cu}_{10^{-}} \text {and } \mathrm{Cu}_{12} \text {-rings }\end{array}$ & $2.587(5)$ & - & - \\
\hline $\begin{array}{l}\mathrm{Cu}^{\cdots} \mathrm{O} \text { interaction range between } \mathrm{Cu}_{8}-\text { and } \\
\mathrm{Cu}_{13} \text {-rings }\end{array}$ & - & $2.386(4)-2.601(5)$ & - \\
\hline $\begin{array}{l}\text { Average of six } \mathrm{Cu}^{\cdots} \cdots \mathrm{O} \text { interactions between } \\
\mathrm{Cu}_{8-} \text { and } \mathrm{Cu}_{13} \text {-rings }\end{array}$ & - & $2.490(4)$ & - \\
\hline $\begin{array}{l}\mathrm{Cu}^{\cdots} \mathrm{O} \text { interaction range between } \mathrm{Cu}_{8}-\text { and } \\
\mathrm{Cu}_{12} \text {-rings }\end{array}$ & - & - & $2.357(7)-2.794(7)$ \\
\hline $\begin{array}{l}\text { Average of six } \mathrm{Cu}^{\cdots} \cdots \mathrm{O} \text { interactions between } \\
\mathrm{Cu}_{8-} \text { and } \mathrm{Cu}_{12} \text {-rings }\end{array}$ & - & - & $2.514(7)$ \\
\hline $\begin{array}{l}\text { Average of all } \mathrm{Cu}^{\cdots} \cdots \mathrm{O} \text { interactions between } \\
\mathrm{Cu}_{n} \text {-rings }\end{array}$ & $2.491(5)$ & $2.490(4)$ & $2.514(4)$ \\
\hline $\begin{array}{l}\text { H-bonded } \mathrm{O} \cdots \mathrm{O} \text { distance range between } \\
\mathrm{Cu}_{6^{-}} \text {and } \mathrm{Cu}_{12} \text {-rings }\end{array}$ & $2.724(5)-2.770(5)$ & - & - \\
\hline $\begin{array}{l}\text { Average of six } \mathrm{H} \text {-bonded } \mathrm{O} \cdots \mathrm{O} \text { distances } \\
\text { between } \mathrm{Cu}_{6}-\text { and } \mathrm{Cu}_{12} \text {-rings }\end{array}$ & $2.754(5)$ & - & - \\
\hline $\begin{array}{l}\text { H-bonded } \mathrm{O} \cdots \mathrm{O} \text { distance range between } \\
\mathrm{Cu}_{10^{-}} \text {and } \mathrm{Cu}_{12} \text {-rings }\end{array}$ & $2.659(7)-2.824(7)$ & - & - \\
\hline $\begin{array}{l}\text { Average of six } \mathrm{H} \text {-bonded } \mathrm{O} \cdots \mathrm{O} \text { distances } \\
\text { between } \mathrm{Cu}_{10-} \text { and } \mathrm{Cu}_{12} \text {-rings }\end{array}$ & $2.753(7)$ & - & - \\
\hline $\begin{array}{l}\mathrm{H} \text {-bonded } \mathrm{O} \cdots \mathrm{O} \text { distance range between } \\
\mathrm{Cu}_{8-} \text { and } \mathrm{Cu}_{13} \text {-rings }\end{array}$ & - & $2.757(5)-2.913(6)$ & - \\
\hline $\begin{array}{l}\text { Average of six } \mathrm{H} \text {-bonded } \mathrm{O} \cdots \mathrm{O} \text { distance } \\
\text { average between } \mathrm{Cu}_{8^{-}} \text {and } \mathrm{Cu}_{13} \text {-rings }\end{array}$ & - & $2.804(6)$ & - \\
\hline $\begin{array}{l}\mathrm{H} \text {-bonded } \mathrm{O} \cdots \mathrm{O} \text { distance range between } \\
\mathrm{Cu}_{8-} \text { and } \mathrm{Cu}_{12} \text {-rings }\end{array}$ & - & - & $2.661(9)-2.768(9)$ \\
\hline $\begin{array}{l}\text { Average of four } \mathrm{H} \text {-bonded } \mathrm{O} \cdots \mathrm{O} \text { distances } \\
\text { between } \mathrm{Cu}_{8^{-}} \text {and } \mathrm{Cu}_{12} \text {-rings }\end{array}$ & - & - & $2.706(9)$ \\
\hline $\begin{array}{l}\text { Average of all } \mathrm{H} \text {-bonded } \mathrm{O} \cdots \mathrm{O} \text { distances } \\
\text { between } \mathrm{Cu}_{\mathrm{n}} \text {-rings }\end{array}$ & $2.754(5)$ & $2.804(6)$ & $2.706(9)$ \\
\hline $\begin{array}{l}\text { H-bonded } \mathrm{O} \cdots \mathrm{O} \text { distance range between } \\
\text { incarcerated ion and } \mathrm{Cu}_{n} \text {-rings }\end{array}$ & $2.748(9)-2.948(9)$ & $2.57(2)-2.92(3)$ & $2.74(2)-3.00(2)$ \\
\hline $\begin{array}{l}\text { Average of all } \mathrm{H} \text {-bonded } \mathrm{O} \cdots \mathrm{O} \text { distances } \\
\text { between incarcerated ion and } \mathrm{Cu}_{\mathrm{n}} \text {-rings }\end{array}$ & $2.822(9)$ & $2.77(2)$ & $2.89(2)$ \\
\hline
\end{tabular}


Table S2. Selected bond lengths for 1. $\mathrm{Cu} 1-\mathrm{Cu}$ : $\mathrm{Cu}_{6}$-ring; $\mathrm{Cu}$ 7-Cu18: $\mathrm{Cu}_{12}$-ring; $\mathrm{Cu}$ 19-Cu28: $\mathrm{Cu}_{10}$-ring.

\begin{tabular}{|c|c|c|c|}
\hline Cu1-O1 1.939(4) & $\mathrm{Cu} 7-\mathrm{N} 13$ 1.952(6) & Cu15-N29 1.975(6) & $\mathrm{Cu} 23-\mathrm{O} 231.931(5)$ \\
\hline Cu1-O6 1.942(4) & $\mathrm{Cu} 7-\mathrm{N} 36$ 1.970(5) & Cu16-O16 1.927(4) & $\mathrm{Cu} 23-\mathrm{N} 44$ 1.941(8) \\
\hline $\mathrm{Cu} 1-\mathrm{N} 12$ 2.006(5) & $\mathrm{Cu} 8-\mathrm{O} 8$ 1.928(4) & Cu16-O15 1.931(4) & $\mathrm{Cu} 23-\mathrm{N} 45$ 1.960(6) \\
\hline Cu1-N1 2.007(5) & $\mathrm{Cu} 8-\mathrm{O} 7$ 1.936(4) & Cu16-N31 1.958(5) & $\mathrm{Cu} 24-\mathrm{N} 47 \mathrm{~B} 1.85(2)$ \\
\hline $\mathrm{Cu} 1-\mathrm{O} 18$ 2.306(4) & Cu8-N14 1.955(5) & Cu16-N30 1.969(5) & $\mathrm{Cu} 24-\mathrm{O} 241.910(5)$ \\
\hline $\mathrm{Cu} 2-\mathrm{O} 1$ 1.934(4) & Cu8-N15 1.980(6) & Cu17-O17 1.931(4) & $\mathrm{Cu} 24-\mathrm{O} 231.925(5)$ \\
\hline $\mathrm{Cu} 2-\mathrm{O} 2$ 1.939(4) & Cu9-O9 1.926(4) & Cu17-O16 1.937(4) & $\mathrm{Cu} 24-\mathrm{N} 46$ 1.965(5) \\
\hline $\mathrm{Cu} 2-\mathrm{N} 3 \mathrm{~B} 1.98(2)$ & Cu9-O8 1.943(4) & Cu17-N33 1.967(5) & $\mathrm{Cu} 24-\mathrm{N} 47$ 2.029(12) \\
\hline $\mathrm{Cu} 2-\mathrm{N} 2$ 1.992(5) & Cu9-N16 1.958(6) & Cu17-N32 1.978(5) & $\mathrm{Cu} 25-\mathrm{N} 48 \mathrm{~B} 1.86(2)$ \\
\hline $\mathrm{Cu} 2-\mathrm{N} 3$ 1.996(11) & Cu9-N17 1.974(6 & $\mathrm{Cu} 18-\mathrm{O} 18$ 1.937(4) & $\mathrm{Cu} 25-\mathrm{O} 25 \mathrm{1.902(4)}$ \\
\hline $\mathrm{Cu} 2-\mathrm{O} 8$ 2.422(4) & Cu10-O9 1.923(4) & Cu18-O17 1.939(4) & $\mathrm{Cu} 25-\mathrm{O} 24$ 1.913(6) \\
\hline $\mathrm{Cu} 3-\mathrm{O} 2$ 1.929(4) & Cu10-O10 1.937(4) & Cu18-N34 1.972(5) & Cu25-N49 1.949(7) \\
\hline $\mathrm{Cu} 3-\mathrm{O} 3$ 1.932(4) & Cu10-N19 1.957(6) & Cu18-N35 1.975(5) & $\mathrm{Cu} 25-\mathrm{N} 48$ 2.007(11) \\
\hline Cu3-N5 1.986(5) & Cu10-N18 1.970(7 & Cu19-O19 1.929(5) & $\mathrm{Cu} 26-\mathrm{O} 261.935(5)$ \\
\hline Cu3-N4 2.002(10) & Cul1-O11 1.927(4) & $\mathrm{Cu} 19-\mathrm{O} 28$ 1.941(4) & $\mathrm{Cu} 26-\mathrm{O} 25$ 1.942(4) \\
\hline $\mathrm{Cu} 3-\mathrm{N} 4 \mathrm{~B} 2.01(2)$ & Cu11-O10 1.929(4) & Cu19-N56 1.991(5) & Cu26-N50 1.988(6) \\
\hline $\mathrm{Cu} 3-\mathrm{O} 102.434(5)$ & Cu11-N21 1.966(5) & Cu19-N37 1.993(6) & Cu26-N51 1.993(5) \\
\hline $\mathrm{Cu} 4-\mathrm{O} 3$ 1.938(4) & Cu11-N20 1.977(6) & $\mathrm{Cu} 20-\mathrm{O} 201.913(5)$ & $\mathrm{Cu} 26-\mathrm{O} 152.364(5)$ \\
\hline $\mathrm{Cu} 4-\mathrm{O} 4$ 1.958(4) & Cu12-O12 1.928(4) & $\mathrm{Cu} 20-\mathrm{O} 191.918(5)$ & $\mathrm{Cu} 27-\mathrm{O} 261.885(5)$ \\
\hline Cu4-N6 1.997(5) & Cu12-O11 1.943(4) & Cu20-N39 1.960(6) & $\mathrm{Cu} 27-\mathrm{O} 27$ 1.902(6) \\
\hline Cu4-N7 2.003(5) & $\mathrm{Cu} 12-\mathrm{N} 23$ 1.975(5) & $\mathrm{Cu} 20-\mathrm{N} 38$ 1.970(6) & $\mathrm{Cu} 27-\mathrm{N} 52$ 1.960(6) \\
\hline $\mathrm{Cu} 4-\mathrm{O} 12$ 2.321(4) & Cu12-N22 1.975(5) & $\mathrm{Cu} 21-\mathrm{O} 21$ 1.924(5) & Cu27-N53 1.964(6) \\
\hline Cu5-O4 1.938(4) & Cu13-O12 1.918(5) & $\mathrm{Cu} 21-\mathrm{O} 201.945(4)$ & $\mathrm{Cu} 28-\mathrm{O} 271.899(5)$ \\
\hline Cu5-O5 1.943(4) & Cu13-O13 1.942(4) & $\mathrm{Cu} 21-\mathrm{N} 41 \mathrm{~B} 1.981(18)$ & $\mathrm{Cu} 28-\mathrm{O} 28$ 1.929(4) \\
\hline Cu5-N9 1.991(5) & Cu13-N25 1.972(5) & $\mathrm{Cu} 21-\mathrm{N} 402.030(7)$ & Cu28-N54 1.977(7) \\
\hline Cu5-N8 2.008(5) & Cu13-N24 1.981(5) & $\mathrm{Cu} 21-\mathrm{N} 412.079(13)$ & $\mathrm{Cu} 28-\mathrm{N} 55$ 1.984(6) \\
\hline $\mathrm{Cu} 5-\mathrm{O} 142.408(4)$ & $\mathrm{Cu} 14-\mathrm{O} 14$ 1.926(4) & $\mathrm{Cu} 21-\mathrm{O} 92.375(6)$ & Se1-O29 1.669(7) \\
\hline Cu6-O5 1.930(4) & Cu14-O13 1.940(4) & $\mathrm{Cu} 22-\mathrm{O} 22$ 1.906(6) & Se1-O30 1.678(8) \\
\hline Cu6-O6 1.937(4) & Cu14-N26 1.974(5) & $\mathrm{Cu} 22-\mathrm{O} 21$ 1.928(4) & Se1-O31 1.695(8) \\
\hline Cu6-N11 1.990(5) & Cu14-N27 1.978(6) & $\mathrm{Cu} 22-\mathrm{N} 42$ 1.942(14) & Se1B-O31B 1.665(15) \\
\hline Cu6-N10 1.991(5) & Cu15-O15 1.924(4) & $\mathrm{Cu} 22-\mathrm{N} 43$ 1.959(7) & Se1B-O30B 1.681(15) \\
\hline $\mathrm{Cu} 7-\mathrm{O} 7$ 1.940(4) & Cu15-O14 1.932(4) & $\mathrm{Cu} 22-\mathrm{N} 42 \mathrm{~B} 1.97(2)$ & Se1B-O29B 1.684(17) \\
\hline $\mathrm{Cu} 7-\mathrm{O} 18$ 1.947(4) & Cu15-N28 1.962(6) & $\mathrm{Cu} 23-\mathrm{O} 221.904(5)$ & \\
\hline
\end{tabular}


Table S3. Selected bond lengths for 2. Cu1-Cu8: Cus-ring; $\mathrm{Cu} 9-\mathrm{Cu} 15$ : $\mathrm{Cu}_{13}$-ring.

\begin{tabular}{|l|l|l|l|}
\hline Cu1-O1 1.937(4) & Cu5-O5 1.886(4) & Cu10-O9 1.909(4) & Cu14-N26 1.962(6) \\
Cu1-O8 1.923(4) & Cu5-O4 1.919(4) & Cu10-O10 1.940(4) & Cu14-O14 1.916(4) \\
Cu1-N1 1.978(5) & Cu5-N8 1.957(5) & Cu10-N19 1.955(5) & Cu14-O13 1.928(4) \\
Cu1-N16 1.982(5) & Cu5-N9 1.962(5) & Cu10-N18 1.982(5) & Cu14-N27 1.964(16) \\
Cu2-O2 1.906(4) & Cu6-O5 1.903(4) & Cu11-O11 1.923(4) & Cu15-N29 1.87(2) \\
Cu2-O1 1.922(4) & Cu6-O6 1.930(4) & Cu11-O10 1.935(4) & Cu15-O14 1.901(4) \\
Cu2-N3 1.986(5) & Cu6-N11 1.983(5) & Cu11-N20 1.988(5) & Cu15-O15 1.912(3) \\
Cu2-N2 1.998(5) & Cu6-N10 1.986(5) & Cu11-N21 1.989(5) & Cu15-N28B 1.92(2) \\
Cu3-O2 1.901(4) & Cu7-O6 1.935(4) & Cu12-N23B 1.87(2) & Cu15-N28 1.993(15) \\
Cu3-O3 1.920(4) & Cu7-O7 1.945(4) & Cu12-O12 1.921(4) & P1-H1P 1.41(12) \\
Cu3-N4 1.982(5) & Cu7-N13 1.979(5) & Cu12-O11 1.922(4) & P1-O16 1.53(2) \\
Cu3-N5B 1.984(5) & Cu7-N12 2.005(5) & Cu12-N22 1.965(6) & P1-O17 1.514(17) \\
Cu3-N5 1.984(5) & Cu7-O12 2.386(4) & Cu12-N23 2.017(13) & P1-O18 1.52(2) \\
Cu4-O3 1.939(4) & Cu8-O8 1.903(4) & Cu13-O13 1.909(4) & P1-O19 1.473(16) \\
Cu4-O4 1.940(4) & Cu8-O7 1.917(4) & Cu13-O12 1.931(4) & P1-O20 1.42(5) \\
Cu4-N6B 1.999(5) & Cu8-N15 1.954(5) & Cu13-N24B 1.96(2) & P1-O21 1.55(5) \\
Cu4-N6 1.999(5) & Cu8-N14 1.965(5) & Cu13-N24 1.958(14) & P1-O22 1.50(2) \\
Cu4-N7 2.027(5) & Cu9-O9 1.926(4) & Cu13-N25 1.965(5) & P1-O23 1.53(4) \\
Cu4-O13 2.392(4) & Cu9-N17 1.959(5) & Cu14-N27B 1.94(2) & P1-O24 1.46(4) \\
\hline
\end{tabular}

Table S4. Selected bond lengths for 3. $\mathrm{Cu} 1-\mathrm{Cu} 8$ : $\mathrm{Cu}_{8}$-ring; $\mathrm{Cu} 9-\mathrm{Cu} 15$ : $\mathrm{Cu}_{12}$-ring.

\begin{tabular}{|l|l|l|l|}
\hline Cu1-O7 1.908(7) & Cu4-N6 1.966(8) & Cu8-O6 1.951(7) & Cu12-N22 1.956(9) \\
Cu1-O8 1.953(6) & Cu4-N7 2.020(8) & Cu8-N14 1.953(8) & Cu12-N23 1.977(9) \\
Cu1-N16 1.991(8) & Cu5-O3 1.902(7) & Cu8-N15 2.009(8) & Cu13-O12 1.928(7) \\
Cu1-N1 1.997(8) & Cu5-O4 1.924(6) & Cu9-O9 1.946(7) & Cu13-O13 1.958(7) \\
Cu1-O9 2.412(7) & Cu5-N8 1.971(8) & Cu9-O9 1.946(7) & Cu13-N24 1.996(9) \\
Cu2-O1 1.885(7) & Cu5-N9 1.976(8) & Cu9-N17 1.989(8) & Cu13-N25 2.004(8) \\
Cu2-O8 1.934(6) & Cu6-O5 1.884(8) & Cu10-O9 1.905(6) & Cu14-O14 1.911(6) \\
Cu2-N2 1.984(8) & Cu6-O4 1.914(6) & Cu10-O10 1.941(7) & Cu14-O13 1.928(7) \\
Cu2-N3 2.014(7) & Cu6-N10 1.956(8) & Cu10-N19 1.961(8) & Cu14-N26 1.957(8) \\
Cu3-O1 1.908(7) & Cu6-N11 1.990(7) & Cu10-N18 1.967(9) & Cu14-N27 1.981(8) \\
Cu3-O2 1.950(6) & Cu7-O5 1.902(8) & Cu11-O11 1.934(6) & Cu15-O14 1.945(7) \\
Cu3-N4 1.989(8) & Cu7-O6 1.943(6) & Cu11-O10 1.946(7) & Cu15-N28 1.980(8) \\
Cu3-N5 2.005(8) & Cu7-N12 1.983(8) & Cu11-N20 1.998(8) & S1-O17 1.417(14) \\
Cu3-O11 2.357(7) & Cu7-N13 2.004(8) & Cu11-N21 1.999(8) & S1-O16 1.482(15) \\
Cu4-O3 1.903(6) & Cu7-O12 2.377(7) & Cu12-O12 1.920(7) & S1-O15 1.489(14) \\
Cu4-O2 1.947(6) & Cu8-O7 1.884(7) & Cu12-O11 1.941(6) & S1-O18 1.507(14) \\
\hline
\end{tabular}


Table S5. Hydrogen bonding data for 1. O1-O6: $\mathrm{Cu}_{6}$-ring; O7-O18: $\mathrm{Cu}_{12}$-ring; O19-O28: $\mathrm{Cu}_{10}$-ring; O29-O31: selenite.

\begin{tabular}{|l|l|l|l|l|}
\hline $\mathbf{D}-\mathbf{H} \cdots \mathbf{A}$ & $\mathbf{D}-\mathbf{H}(\mathbf{\AA})$ & $\mathbf{H} \cdots \mathbf{A}(\mathbf{\AA})$ & $\mathbf{D} \cdots \mathbf{A}(\mathbf{A})$ & $\mathbf{D}-\mathbf{H} \cdots \mathbf{A}\left({ }^{\circ}\right)$ \\
\hline $\mathrm{O} 1-\mathrm{H} 1 \mathrm{O} \cdots \mathrm{O} 30$ & $0.83(2)$ & $1.97(3)$ & $2.787(9)$ & $166(7)$ \\
\hline $\mathrm{O} 2-\mathrm{H} 2 \mathrm{O} \cdots \mathrm{O} 30$ & $0.83(2)$ & $2.65(3)$ & $3.469(9)$ & $167(7)$ \\
\hline $\mathrm{O} 2-\mathrm{H} 2 \mathrm{O} \cdots \mathrm{O} 29 \mathrm{~B}$ & $0.83(2)$ & $1.95(4)$ & $2.744(17)$ & $159(7)$ \\
\hline $\mathrm{O} 3-\mathrm{H} 3 \mathrm{O} \cdots \mathrm{O} 29$ & $0.84(2)$ & $1.94(2)$ & $2.775(9)$ & $173(7)$ \\
\hline $\mathrm{O} 3-\mathrm{H} 3 \mathrm{O} \cdots \mathrm{O} 29 \mathrm{~B}$ & $0.84(2)$ & $2.60(5)$ & $3.286(18)$ & $140(6)$ \\
\hline $\mathrm{O} 4-\mathrm{H} 4 \mathrm{O} \cdots \mathrm{O} 3$ & $0.85(2)$ & $2.66(4)$ & $3.387(10)$ & $145(6)$ \\
\hline $\mathrm{O} 4-\mathrm{H} 4 \mathrm{O} \cdots \mathrm{O} 31 \mathrm{~B}$ & $0.85(2)$ & $2.12(5)$ & $2.801(15)$ & $137(6)$ \\
\hline $\mathrm{O} 5-\mathrm{H} 5 \mathrm{O} \cdots \mathrm{O} 3$ & $0.84(2)$ & $1.91(2)$ & $2.748(9)$ & $172(7)$ \\
\hline $\mathrm{O} 6-\mathrm{H} 6 \mathrm{O} \cdots \mathrm{O} 3$ & $0.84(2)$ & $2.50(4)$ & $3.242(9)$ & $148(6)$ \\
\hline $\mathrm{O} 6-\mathrm{H} 6 \mathrm{O} \cdots \mathrm{O} 30 \mathrm{~B}$ & $0.84(2)$ & $1.98(4)$ & $2.751(15)$ & $153(7)$ \\
\hline $\mathrm{O} 7-\mathrm{H} 7 \mathrm{O} \cdots \mathrm{O} 1$ & $0.82(2)$ & $1.99(2)$ & $2.767(5)$ & $159(5)$ \\
\hline $\mathrm{O} 9-\mathrm{H} 9 \mathrm{O} \cdots \mathrm{O} 2$ & $0.84(2)$ & $1.92(3)$ & $2.747(6)$ & $168(8)$ \\
\hline $\mathrm{O} 10-\mathrm{H} 10 \mathrm{O} \cdots \mathrm{O} 21$ & $0.84(2)$ & $1.98(3)$ & $2.815(8)$ & $171(9)$ \\
\hline $\mathrm{O} 11-\mathrm{H} 11 \mathrm{O} \cdots \mathrm{O} 3$ & $0.84(2)$ & $2.03(5)$ & $2.752(5)$ & $145(7)$ \\
\hline $\mathrm{O} 12-\mathrm{H} 12 \mathrm{O} \cdots \mathrm{O} 23$ & $0.84(2)$ & $1.90(4)$ & $2.659(7)$ & $149(7)$ \\
\hline $\mathrm{O} 13-\mathrm{H} 13 \mathrm{O} \cdots \mathrm{O} 4$ & $0.83(2)$ & $1.96(3)$ & $2.764(5)$ & $167(7)$ \\
\hline $\mathrm{O} 14-\mathrm{H} 14 \mathrm{O} \cdots \mathrm{O} 5$ & $0.83(2)$ & $2.53(5)$ & $3.186(5)$ & $136(6)$ \\
\hline $\mathrm{O} 14-\mathrm{H} 14 \mathrm{O} \cdots \mathrm{O} 25$ & $0.83(2)$ & $2.18(6)$ & $2.773(7)$ & $129(6)$ \\
\hline $\mathrm{O} 15-\mathrm{H} 15 \mathrm{O} \cdots \mathrm{O} 5$ & $0.84(2)$ & $1.90(3)$ & $2.724(5)$ & $167(8)$ \\
\hline $\mathrm{O} 16-\mathrm{H} 16 \mathrm{O} \cdots \mathrm{O} 26$ & $0.84(2)$ & $2.03(4)$ & $2.824(7)$ & $158(7)$ \\
\hline $\mathrm{O} 17-\mathrm{H} 17 \mathrm{O} \cdots \mathrm{O} 6$ & $0.84(2)$ & $1.97(3)$ & $2.770(5)$ & $159(8)$ \\
\hline $\mathrm{O} 18-\mathrm{H} 18 \mathrm{O} \cdots \mathrm{O} 28$ & $0.83(2)$ & $1.86(3)$ & $2.681(7)$ & $169(7)$ \\
\hline $\mathrm{O} 19-\mathrm{H} 19 \mathrm{O} \cdots \mathrm{O} 30$ & $0.83(2)$ & $2.24(7)$ & $2.843(9)$ & $130(8)$ \\
\hline $\mathrm{O} 21-\mathrm{H} 21 \mathrm{O} \cdots \mathrm{O} 29$ & $0.84(2)$ & $2.65(7)$ & $3.190(10)$ & $124(7)$ \\
\hline $\mathrm{O} 21-\mathrm{H} 21 \mathrm{O} \cdots \mathrm{O} 29 \mathrm{~B}$ & $0.84(2)$ & $2.06(5)$ & $2.830(17)$ & $151(8)$ \\
\hline $\mathrm{O} 22-\mathrm{H} 22 \mathrm{O} \cdots \mathrm{O} 29$ & $0.84(2)$ & $2.18(8)$ & $2.797(10)$ & $130(6)$ \\
\hline $\mathrm{O} 23-\mathrm{H} 23 \mathrm{O} \cdots \mathrm{O} 29$ & $0.84(2)$ & $2.28(6)$ & $2.907(9)$ & $131(7)$ \\
\hline
\end{tabular}


Table S6. Hydrogen bonding data for 2. O1-O8: $\mathrm{Cu}_{8}$-ring; O9-O15: $\mathrm{Cu}_{13}$-ring; O16-O24: phosphite. Symmetry code: (i) $-\mathrm{x}+1, \mathrm{y},-\mathrm{z}+3 / 2$.

\begin{tabular}{|c|c|c|c|c|}
\hline$D-\mathrm{H} \cdots A$ & $D-\mathrm{H}(\AA)$ & $\mathrm{H} \cdots A(\AA)$ & $D \cdots A(\AA)$ & $D-\mathrm{H}^{\cdots} A\left(^{\circ}\right)$ \\
\hline $\mathrm{O} 1-\mathrm{H} 1 \mathrm{O} \cdots \mathrm{O} 17 \mathrm{~b}$ & $0.83(2)$ & $2.14(4)$ & $2.928(16)$ & $158(7)$ \\
\hline $\mathrm{O} 1-\mathrm{H} 1 \mathrm{O} \cdots \mathrm{O} 17 \mathrm{~b}^{\mathrm{i}}$ & $0.83(2)$ & $1.91(3)$ & $2.726(16)$ & $167(7)$ \\
\hline $\mathrm{O} 1-\mathrm{H} 1 \mathrm{O} \cdots \mathrm{O} 19 \mathrm{c}$ & $0.83(2)$ & $1.84(3)$ & $2.667(13)$ & $172(7)$ \\
\hline $\mathrm{O} 1-\mathrm{H} 1 \mathrm{O} \cdots \mathrm{O} 24 \mathrm{~d}^{\mathrm{i}}$ & $0.83(2)$ & $2.25(4)$ & $3.03(3)$ & $156(7)$ \\
\hline $\mathrm{O} 2-\mathrm{H} 2 \mathrm{O} \cdots \mathrm{O} 16 \mathrm{~b}$ & $0.82(2)$ & $2.24(4)$ & $3.02(19)$ & $158(8)$ \\
\hline $\mathrm{O} 2-\mathrm{H} 2 \mathrm{O} \cdots \mathrm{O} 17 \mathrm{~b}^{\mathrm{i}}$ & $0.82(2)$ & $2.41(6)$ & $3.058(17)$ & $136(7)$ \\
\hline $\mathrm{O} 2-\mathrm{H} 2 \mathrm{O} \cdots \mathrm{O} 23 \mathrm{~d}$ & $0.82(2)$ & $2.08(6)$ & $2.82(3)$ & $148(8)$ \\
\hline $\mathrm{O} 2-\mathrm{H} 2 \mathrm{O} \cdots \mathrm{O} 24 \mathrm{~d}^{\mathrm{i}}$ & $0.82(2)$ & $2.11(6)$ & $2.81(3)$ & $143(7)$ \\
\hline $\mathrm{O} 3-\mathrm{H} 3 \mathrm{O} \cdots \mathrm{O} 16 \mathrm{~b}$ & $0.83(2)$ & $1.86(3)$ & $2.675(16)$ & $165(7)$ \\
\hline $\mathrm{O} 3-\mathrm{H} 3 \mathrm{O} \cdots \mathrm{O} 18 \mathrm{~b}^{\mathrm{i}}$ & $0.83(2)$ & $2.44(5)$ & $3.140(18)$ & $142(7)$ \\
\hline $\mathrm{O} 3-\mathrm{H} 3 \mathrm{O} \cdots \mathrm{O} 20 \mathrm{c}^{\mathrm{i}}$ & $0.83(2)$ & $2.06(6)$ & $2.80(5)$ & $149(7)$ \\
\hline $\mathrm{O} 3-\mathrm{H} 3 \mathrm{O} \cdots \mathrm{O} 21 \mathrm{c}$ & $0.83(2)$ & 2.02(6) & $2.79(5)$ & $155(7)$ \\
\hline $\mathrm{O} 3-\mathrm{H} 3 \mathrm{O} \cdots \mathrm{O} 23 \mathrm{~d}$ & $0.83(2)$ & $2.19(4)$ & $3.00(3)$ & $167(7)$ \\
\hline $\mathrm{O} 3-\mathrm{H} 3 \mathrm{O} \cdots \mathrm{O} 24 \mathrm{~d}^{\mathrm{i}}$ & $0.83(2)$ & $2.64(4)$ & $3.44(3)$ & $160(7)$ \\
\hline $\mathrm{O} 4-\mathrm{H} 4 \mathrm{O} \cdots \mathrm{O} 16 \mathrm{~b}$ & $0.83(2)$ & 2.46(3) & $3.259(18)$ & $163(7)$ \\
\hline $\mathrm{O} 4-\mathrm{H} 4 \mathrm{O} \cdots \mathrm{O} 18 \mathrm{~b}^{\mathrm{i}}$ & $0.83(2)$ & $1.86(4)$ & $2.645(16)$ & $158(7)$ \\
\hline $\mathrm{O} 4-\mathrm{H} 4 \mathrm{O} \cdots \mathrm{O} 20 \mathrm{c}^{\mathrm{i}}$ & $0.83(2)$ & $1.95(4)$ & $2.76(2)$ & $166(7)$ \\
\hline $\mathrm{O} 4-\mathrm{H} 4 \mathrm{O} \cdots \mathrm{O} 21 \mathrm{c}$ & $0.83(2)$ & 2.13(4) & 2.92(3) & $160(7)$ \\
\hline $\mathrm{O} 4-\mathrm{H} 4 \mathrm{O} \cdots \mathrm{O} 22 \mathrm{~d}$ & $0.83(2)$ & $2.39(6)$ & $3.08(3)$ & $142(6)$ \\
\hline $\mathrm{O} 4-\mathrm{H} 4 \mathrm{O} \cdots \mathrm{O} 22 \mathrm{~d}^{\mathrm{i}}$ & $0.83(2)$ & $2.03(5)$ & $2.77(3)$ & $147(7)$ \\
\hline $\mathrm{O} 5-\mathrm{H} 5 \mathrm{O} \cdots \mathrm{O} 18 \mathrm{~b}$ & $0.84(2)$ & $1.97(5)$ & $2.742(18)$ & $152(7)$ \\
\hline $\mathrm{O} 5-\mathrm{H} 5 \mathrm{O} \cdots \mathrm{O} 18 \mathrm{~b}^{\mathrm{i}}$ & $0.84(2)$ & $2.47(5)$ & $3.23(2)$ & $153(7)$ \\
\hline $\mathrm{O} 5-\mathrm{H} 5 \mathrm{O} \cdots \mathrm{O} 20 \mathrm{c}$ & $0.84(2)$ & $2.39(6)$ & $3.14(4)$ & $150(7)$ \\
\hline $\mathrm{O} 5-\mathrm{H} 5 \mathrm{O} \cdots \mathrm{O} 21 \mathrm{c}^{\mathrm{i}}$ & $0.84(2)$ & $2.36(7)$ & $3.10(4)$ & $147(7)$ \\
\hline $\mathrm{O} 5-\mathrm{H} 5 \mathrm{O} \cdots \mathrm{O} 22 \mathrm{~d}$ & $0.84(2)$ & $1.88(6)$ & $2.67(4)$ & $156(8)$ \\
\hline $\mathrm{O} 5-\mathrm{H} 5 \mathrm{O} \cdots \mathrm{O} 22 \mathrm{~d}^{\mathrm{i}}$ & $0.84(2)$ & $1.97(6)$ & $2.76(4)$ & $156(8)$ \\
\hline $\mathrm{O} 6-\mathrm{H} 6 \mathrm{O} \cdots \mathrm{O} 16 \mathrm{~b}^{\mathrm{i}}$ & $0.82(2)$ & 2.11(3) & $2.917(17)$ & $166(7)$ \\
\hline $\mathrm{O} 6-\mathrm{H} 6 \mathrm{O} \cdots \mathrm{O} 18 \mathrm{~b}$ & $0.82(2)$ & 2.01(4) & $2.79(2)$ & $158(7)$ \\
\hline $\mathrm{O} 6-\mathrm{H} 6 \mathrm{O} \cdots \mathrm{O} 20 \mathrm{c}$ & $0.82(2)$ & $1.96(4)$ & $2.78(3)$ & $173(7)$ \\
\hline $\mathrm{O} 6-\mathrm{H} 6 \mathrm{O} \cdots \mathrm{O} 21 \mathrm{c}^{\mathrm{i}}$ & $0.82(2)$ & $1.76(3)$ & $2.57(2)$ & $171(8)$ \\
\hline $\mathrm{O} 6-\mathrm{H} 6 \mathrm{O} \cdots \mathrm{O} 22 \mathrm{~d}$ & $0.82(2)$ & $2.60(5)$ & $3.33(4)$ & $149(7)$ \\
\hline $\mathrm{O} 6-\mathrm{H} 6 \mathrm{O} \cdots \mathrm{O} 23 \mathrm{~d}^{\mathrm{i}}$ & $0.82(2)$ & $2.54(5)$ & $3.30(3)$ & $153(7)$ \\
\hline $\mathrm{O} 7-\mathrm{H} 7 \mathrm{O} \cdots \mathrm{O}^{2} 6 \mathrm{~b}^{\mathrm{i}}$ & $0.84(2)$ & $2.08(4)$ & 2.846(19) & $152(7)$ \\
\hline $\mathrm{O} 7-\mathrm{H} 7 \mathrm{O} \cdots \mathrm{O} 17 \mathrm{~b}$ & $0.84(2)$ & $2.60(5)$ & $3.331(16)$ & $147(7)$ \\
\hline
\end{tabular}




\begin{tabular}{|l|l|l|l|l|}
\hline $\mathrm{O} 7-\mathrm{H} 7 \mathrm{O} \cdots \mathrm{O} 20 \mathrm{c}$ & $0.84(2)$ & $2.56(6)$ & $3.32(4)$ & $151(7)$ \\
\hline $\mathrm{O} 7-\mathrm{H} 7 \mathrm{O} \cdots \mathrm{O} 21 \mathrm{c}^{\mathrm{i}}$ & $0.84(2)$ & $2.26(6)$ & $3.03(4)$ & $152(7)$ \\
\hline $\mathrm{O} 7-\mathrm{H} 7 \mathrm{O} \cdots \mathrm{O} 23 \mathrm{~d}^{\mathrm{i}}$ & $0.84(2)$ & $1.87(5)$ & $2.64(3)$ & $153(7)$ \\
\hline $\mathrm{O} 7-\mathrm{H} 7 \mathrm{O} \cdots \mathrm{O} 24 \mathrm{~d}$ & $0.84(2)$ & $2.17(5)$ & $2.92(3)$ & $150(7)$ \\
\hline $\mathrm{O} 8-\mathrm{H} 8 \mathrm{O} \cdots \mathrm{O} 17 \mathrm{~b}$ & $0.82(2)$ & $1.86(3)$ & $2.656(15)$ & $163(7)$ \\
\hline $\mathrm{O} 8-\mathrm{H} 8 \mathrm{O} \cdots \mathrm{O} 19 \mathrm{c}$ & $0.82(2)$ & $2.28(4)$ & $3.019(9)$ & $150(7)$ \\
\hline $\mathrm{O} 8-\mathrm{H} 8 \mathrm{O} \cdots \mathrm{O} 23 \mathrm{~d}^{\mathrm{i}}$ & $0.82(2)$ & $2.20(4)$ & $3.00(3)$ & $165(7)$ \\
\hline $\mathrm{O} 8-\mathrm{H} 8 \mathrm{O} \cdots \mathrm{O} 24 \mathrm{~d}$ & $0.82(2)$ & $1.94(4)$ & $2.76(3)$ & $174(8)$ \\
\hline $\mathrm{O} 9-\mathrm{H} 9 \mathrm{O} \cdots \mathrm{O} 1$ & $0.83(2)$ & $1.95(3)$ & $2.757(5)$ & $163(7)$ \\
\hline $\mathrm{O} 10-\mathrm{H} 10 \mathrm{O} \cdots \mathrm{O} 8^{\mathrm{i}}$ & $0.84(2)$ & $2.11(3)$ & $2.913(6)$ & $160(7)$ \\
\hline $\mathrm{O} 11-\mathrm{H} 11 \mathrm{O} \cdots \mathrm{O} 7^{\mathrm{i}}$ & $0.83(2)$ & $1.98(3)$ & $2.803(6)$ & $169(7)$ \\
\hline $\mathrm{O} 12-\mathrm{H} 12 \mathrm{O} \cdots \mathrm{O} 3$ & $0.83(2)$ & $1.97(3)$ & $2.784(5)$ & $164(7)$ \\
\hline $\mathrm{O} 13-\mathrm{H} 13 \mathrm{O} \cdots \mathrm{O} 6^{\mathrm{i}}$ & $0.83(2)$ & $1.95(3)$ & $2.767(6)$ & $169(8)$ \\
\hline $\mathrm{O} 14-\mathrm{H} 14 \mathrm{O} \cdots \mathrm{O} 4$ & $0.83(2)$ & $1.98(3)$ & $2.802(6)$ & $168(8)$ \\
\hline $\mathrm{O} 15-\mathrm{H} 15 \mathrm{O} \cdots \mathrm{O} 5$ & $0.84(2)$ & $2.51(2)$ & $3.337(6)$ & $168(10)$ \\
\hline
\end{tabular}


Table S7. Hydrogen bonding data for 3. O1-O8: $\mathrm{Cu}_{8}$-ring; O9-O14: $\mathrm{Cu}_{12}$-ring; O15-O18: sulfate. Symmetry code: (i) $-\mathrm{x}+1,-\mathrm{y}+1, \mathrm{z}$.

\begin{tabular}{|l|l|l|l|l|}
\hline$D-\mathrm{H} \cdots A$ & $D-\mathrm{H}(\AA)$ & $\mathrm{H} \cdots A(\AA)$ & $D \cdots A(\AA)$ & $D-\mathrm{H}^{\cdots} A\left(^{\circ}\right)$ \\
\hline $\mathrm{O} 1-\mathrm{H} 1 \mathrm{O} \cdots \mathrm{O} 15 \mathrm{a}$ & $0.83(3)$ & $2.30(3)$ & $3.026(18)$ & $145(6)$ \\
\hline $\mathrm{O} 1-\mathrm{H} 1 \mathrm{O} \cdots \mathrm{O} 17 \mathrm{a}^{\mathrm{i}}$ & $0.83(3)$ & $1.99(5)$ & $2.787(16)$ & $160(13)$ \\
\hline $\mathrm{O} 2-\mathrm{H} 2 \mathrm{O} \cdots \mathrm{O} 17 \mathrm{a}^{\mathrm{i}}$ & $0.82(3)$ & $2.10(4)$ & $2.898(16)$ & $163(11)$ \\
\hline $\mathrm{O} 2-\mathrm{H} 2 \mathrm{O} \cdots \mathrm{O} 18 \mathrm{a}$ & $0.82(3)$ & $2.35(7)$ & $3.078(17)$ & $147(10)$ \\
\hline $\mathrm{O} 3-\mathrm{H} 3 \mathrm{O} \cdots \mathrm{O} 17 \mathrm{a}^{\mathrm{i}}$ & $0.84(3)$ & $2.20(5)$ & $3.003(18)$ & $161(11)$ \\
\hline $\mathrm{O} 3-\mathrm{H} 3 \mathrm{O} \cdots \mathrm{O} 18 \mathrm{a}$ & $0.84(3)$ & $2.10(7)$ & $2.850(15)$ & $148(11)$ \\
\hline $\mathrm{O} 4-\mathrm{H} 4 \mathrm{O} \cdots \mathrm{O} 16 \mathrm{a}$ & $0.83(3)$ & $2.61(5)$ & $3.408(17)$ & $161(11)$ \\
\hline $\mathrm{O} 4-\mathrm{H} 4 \mathrm{O} \cdots \mathrm{O} 18 \mathrm{a}$ & $0.83(3)$ & $2.51(8)$ & $3.212(18)$ & $143(10)$ \\
\hline $\mathrm{O} 4-\mathrm{H} 4 \mathrm{O} \cdots \mathrm{O} 18 \mathrm{a}^{\mathrm{i}}$ & $0.83(3)$ & $2.05(7)$ & $2.780(16)$ & $147(11)$ \\
\hline $\mathrm{O} 5-\mathrm{H} 5 \mathrm{O} \cdots \mathrm{O} 16 \mathrm{a}$ & $0.87(2)$ & $2.04(2)$ & $2.901(16)$ & $172(14)$ \\
\hline $\mathrm{O} 5-\mathrm{H} 5 \mathrm{O} \cdots \mathrm{O} 18 \mathrm{a}^{\mathrm{i}}$ & $0.87(2)$ & $2.26(11)$ & $2.925(17)$ & $134(12)$ \\
\hline $\mathrm{O} 6-\mathrm{H} 6 \mathrm{O} \cdots \mathrm{O} 15 \mathrm{a}^{\mathrm{i}}$ & $0.81(3)$ & $2.24(6)$ & $2.979(17)$ & $152(11)$ \\
\hline $\mathrm{O} 6-\mathrm{H} 6 \mathrm{O} \cdots \mathrm{O} 16 \mathrm{a}$ & $0.81(3)$ & $2.17(5)$ & $2.937(16)$ & $159(11)$ \\
\hline $\mathrm{O} 7-\mathrm{H} 7 \mathrm{O} \cdots \mathrm{O} 15 \mathrm{a}^{\mathrm{i}}$ & $0.84(3)$ & $2.17(7)$ & $2.934(17)$ & $151(13)$ \\
\hline $\mathrm{O} 8-\mathrm{H} 8 \mathrm{O} \cdots \mathrm{O} 15 \mathrm{a}$ & $0.84(3)$ & $1.94(5)$ & $2.739(16)$ & $157(10)$ \\
\hline $\mathrm{O} 8-\mathrm{H} 8 \mathrm{O} \cdots \mathrm{O} 15 \mathrm{a}^{\mathrm{i}}$ & $0.84(3)$ & $2.57(7)$ & $3.32(2)$ & $149(10)$ \\
\hline $\mathrm{O} 8-\mathrm{H} 8 \mathrm{O} \cdots \mathrm{O} 17 \mathrm{a}^{\mathrm{i}}$ & $0.84(3)$ & $2.57(6)$ & $3.338(18)$ & $152(10)$ \\
\hline $\mathrm{O} 9-\mathrm{H} 9 \mathrm{O} \cdots \mathrm{O} 8$ & $0.85(3)$ & $1.87(3)$ & $2.709(9)$ & $173(12)$ \\
\hline $\mathrm{O} 11-\mathrm{H} 110 \cdots \mathrm{O} 6{ }^{\mathrm{i}}$ & $0.86(3)$ & $1.85(4)$ & $2.686(9)$ & $163(11)$ \\
\hline $\mathrm{O} 12-\mathrm{H} 12 \mathrm{O} \cdots \mathrm{O} 2$ & $0.86(3)$ & $1.87(6)$ & $2.660(9)$ & $153(11)$ \\
\hline $\mathrm{O} 14-\mathrm{H} 140 \cdots \mathrm{O} 4{ }^{\mathrm{i}}$ & $0.86(3)$ & $2.03(9)$ & $2.768(9)$ & $147(11)$ \\
\hline
\end{tabular}

